Cahiers d'études africaines

CHRONIQUE BIBLIOGRAPHIQUE

\title{
Voyage en Brejnevie
}

Vie « rêvée » des étudiants du Tiers-monde en Russie soviétique

Jean-José Maboungou

\section{OpenEdition \\ Journals}

Édition électronique

URL : http://journals.openedition.org/etudesafricaines/20755

DOI : 10.4000 /etudesafricaines.20755

ISSN : $1777-5353$

Éditeur

Éditions de l'EHESS

\section{Édition imprimée}

Date de publication : 1 juillet 2017

Pagination : 445-452

ISBN : 978-2-7132-2688-5

ISSN : 0008-0055

Référence électronique

Jean-José Maboungou, « Voyage en Brejnevie », Cahiers d'études africaines [En ligne], 226 | 2017, mis en ligne le 01 avril 2019, consulté le 02 janvier 2020. URL : http://journals.openedition.org/ etudesafricaines/20755; DOI : 10.4000/etudesafricaines.20755 


\title{
Voyage en Brejnevie
}

\author{
Vie « rêvée » des étudiants du Tiers-monde \\ en Russie soviétique*
}

Au début des années 1960, d'importants contingents d'étudiants africains issus de pays se réclamant de "l'expérience socialiste », de l'anti-impérialisme (Égypte, Algérie, Tanzanie, Congo-Brazzaville, Mali, Guinée, Ghana, etc.), et de mouvements de libération nationale (ANC, MPLA, FRELIMO, SWAPO, etc.) furent envoyés étudier en URSS et dans les démocraties populaires, ainsi qu'en Chine populaire et à Cuba. Quelles expériences ces étudiants ont-ils vécues dans ces pays ? Quel a été leur quotidien, notamment dans l'Union soviétique des années 1970, pays qu'ils connaissaient, certes, à travers l'actualité internationale, les cours d'histoire ou les lectures individuelles, mais qui était si éloigné de leur culture d'origine ?

Deux livres tentent de répondre à ces questions à partir notamment de ce que leurs auteurs ont vécu personnellement en URSS : Un Africain dans un iceberg. Impossible amour à Saint-Pétersbourg de Zounga Bongolo et Le Gel de Sonallah Ibrahim.

Le premier auteur, né à Brazzaville en 1955, a étudié en URSS de 1973 à 1978, à l'Institut pédagogique Herzen de Leningrad où il obtient un Master en philologie qui lui permet d'enseigner la langue russe et la philosophie au Lycée technique de Pointe-Noire. En 1982, il repart à Leningrad pour une durée de trois ans en vue d'un doctorat de philosophie, option communisme scientifique, qu'il obtient en 1985 à la faveur des bouleversements politiques ayant suivi la chute du mur de Berlin en 1989 et du processus de démocratisation en Afrique avec les Conférences nationales. Il fonde l'un des premiers journaux libres du pays, La Rue meurt, qu'il a dirigé jusqu'à une date récente.

Le second est un homme de gauche qui a connu la prison sous le régime de Nasser, et que l'on cite parmi les grands écrivains de l'Égypte contemporaine. Neuf de ses romans, traduits de l'arabe en français, ont été publiés chez Actes Sud. À l'instar de Zounga Bongolo, Sonallah Ibrahim a séjourné comme étudiant en URSS dans les années 1970.

À propos des livres : Zounga Bongolo, Un Africain dans un iceberg. Impossible amour à Saint-Pétersbourg, Brazzaville-Paris, Éditions Paari, 2006, 157 p. ; Ibrahim SonAlLAH, Le Gel, traduit de l'arabe (Égypte) par Richard Jacquemond, Arles, Actes Sud-Sindbad, 2015, 309 p. 
Écrit autour de 1983, Un Africain dans un iceberg n'a été publié qu'en 2006. Le contexte idéologique de l'époque laisse penser à une autocensure, car publier un roman critique à l'endroit de l'URSS dans un pays se réclamant du marxisme-léninisme, eut été s'exposer certainement à des sanctions.

C'est probablement l'assassinat à Saint-Pétersbourg, en avril 2006, de l'étudiant sénégalais Lampsar Sall, selon toute vraisemblance par un groupe néo-nazi, qui a motivé Zounga Bongolo à publier le livre, en réaction à ce crime raciste. Ce livre est dédié à cet étudiant ainsi qu'à Mikhaïl Gorbatchev pour son rôle en faveur de la perestroïka.

Un Africain dans un iceberg est un roman dont le propos retrace l'histoire d'un amour impossible entre Jan et Natacha, une jeune Soviétique originaire de Narva en Estonie. Tous deux sont étudiants à l'Institut pédagogique d'État « Herzen » de Leningrad ; lui, en faculté de sciences politiques, elle, en faculté de physique. Logés à leur arrivée dans la même cité universitaire, Jan et Natacha apprennent à se connaître et à s'apprécier, et au fil du temps tombent amoureux l'un de l'autre. Mais la mère de Natacha vit cette idylle comme une trahison, et elle n'est pas la seule : les condisciples de Natacha à l'Institut, ses professeurs, ses amis, le lui font savoir ouvertement, sans ambages; «filer le bel amour » avec un «Gospadine niègre » ${ }^{1}$, est indigne d'une jeune fille soviétique de bonne éducation.

En 1974, année où Jan et Natacha se lient d'amitié, le «fond raciste » de la société soviétique était vivace; il n'était pas rare en effet qu'un Noir se fasse traiter de « Niègre » ou de « singe noir » en dehors de sa cité universitaire, même dans des villes ouvertes à la culture et à la tolérance comme Moscou ou Leningrad. Dans ce climat, Natacha doit faire face à une famille de plus en plus réprobatrice. Ses proches, telle Tyotia Zoïa (tante Zoïa, une amie de la mère de Natacha) laissent éclater leur colère : "Oh Gospadi ${ }^{2}$ ! Est-ce de la malédiction? Une tentation ? [...] Où est donc ta conscience ? Où est ton intelligence, ton honneur de femme soviétique, de communiste ? » (pp. 36-37). Le drame que vit Natacha n'est pourtant pas unique en son genre. La grande majorité des filles qui entretiennent des liaisons avec des Africains sont logées à la même enseigne.

Sommée par le doyen d'interrompre sa liaison sous peine d'être exclue de son logement étudiant (p. 102), Natacha paie finalement très cher son amour «irraisonné » pour Jan. Elle est radiée du Komsomol (Union de la jeunesse socialiste soviétique) pour conduite immorale, ce qui entrâne de facto son exclusion de l'Institut tandis que sa mère, qui s'était farouchement opposée à son mariage, se suicide par pendaison, à la suite d'un accès dépressif.

Zounga Bongolo tente d'absoudre le geste de la mère en décrivant l'entre-deux inconfortable dans lequel elle se situe, entre la pression sociale et l'amour pour sa fille. Son opposition au mariage de Natacha avec Jan

1. «Monsieur le Nègre », p. 28.

2. «Mon Dieu!», p. 37. 
ne s'expliquerait pas par une réaction raciste de sa part. Peut-être cette mère, pour qui la réussite professionnelle et sociale de sa fille comptait par-dessus tout, cherchait tout simplement à la protéger du racisme ambiant sous le poids duquel elle allait elle-même succomber. Ce que semble confirmer cet échange avec sa fille, enceinte de Jan (mais contrainte d'avorter) :

[...] As-tu pensé à son avenir ? Tu aurais accouché d'un bâtard apatride qui t'enfoncerait dans l'abîme. Ce serait ton fardeau de la vie, lourd à porter. Dans les magasins, à l'hôpital, dans le métro, tout le monde se retournerait vers toi et ton enfant de couleur, en te traitant de chipie de Nègres. T'imagines-tu quelle honte! Tes propres amis ne t'ouvriraient plus leur porte. [...] Les portes pour un travail honorable te seraient fermées au nez avec des formules de politesse dès que l'on découvrirait ton Négrillon. [...] La conscience populaire demeure réticente au métissage. La majorité des femmes qui ont osé braver cet ordre ne sont devenues que des infortunées exposées à la risée publique. J'en ai vu une que l'on conspuait dans un magasin. Le soir, elle rentra chez elle et étouffa son mulâtre jusqu'à l'asphyxie. Puis, elle se présenta de son chef à la milice (pp. 140-141).

Quant à Jan, contraint d'admettre, non sans une certaine amertume, qu'il n'était pas destiné à Natacha, en raison tout simplement de la couleur de sa peau, il n'en conçoit pas pour autant une quelconque forme de ressentiment. Coupable de s'obstiner à aimer cette fille, sa parole est un cri d'abandon qui sonne comme un aveu de lucidité. Cette lucidité à laquelle il se résigne laisse entrevoir qu'il était venu en Union soviétique pour étudier, et lui vient de cet exercice dialectique auquel se livre Natacha dans une espèce de monologue :

On avait souvent tort de penser que, n'ayant pratiqué ni l'esclavage ni la colonisation, le peuple russe serait plus disposé que celui de l'Occident à promouvoir la civilisation de l'Universel par la fusion des races et de leurs cultures. [...] par manque de contact direct avec le Noir, tous les témoignages rapportés de l'esclavage ou de la colonisation ont été acceptés crûment, de façon métaphysique, sans éléments de comparaison et d'appréciation graduelle, progressive dans le temps (p. 93).

Jusqu'à la fin du second conflit mondial, l'Union soviétique était restée largement fermée à l'Occident et à de vastes régions du monde. Même si de nombreux militants africains, communistes et anticolonialistes avaient séjourné dans les écoles de formation du Kominterm ou du Kominform, si une grande université portait le nom du grand leader africain Patrice Lumumba, et si l'Institut d'Afrique de l'Académie des sciences de l'uRss, fondé en 1959, avait publié jusqu'en 1974 plus de 620 ouvrages sur l'Afrique ${ }^{3}$, il faut croire que les rapports entre l'Afrique et l'Union soviétique étaient plutôt récents. En 1974, nombreux étaient encore les Soviétiques qui n'avaient jamais rencontré un Noir. C'est à la faveur du mouvement des Indépendances en

3. C. WAUTHIER, «Les études africaines en Union soviétique. Une interprétation du colonialisme, du racisme et du développement », Le Monde diplomatique, mai 1976, p. 21. 
Afrique et des luttes de libération nationale à travers le monde que la patrie du socialisme allait vraiment s'ouvrir à l'Afrique noire. Ceci explique dans une large mesure qu'elle soit demeurée sous l'influence des préjugés racistes légués par les écrits coloniaux qui avaient valeur d'objectivité dans un pays qui se revendiquait pourtant de l'Internationalisme. Le déficit de connaissance des Africains dans un pays fraîchement industrialisé et encore marqué de son vieux fond moujik à tous les niveaux de l'échelle sociale, y compris à celui des élites, ne pouvait qu'alimenter dans la population soviétique un racisme diffus avec son chapelet d'idées reçues sur la soi-disant « inconsistance » de la race noire; lequel racisme, on le sait, pouvait se manifester sous des formes d'une extrême violence.

Il convient peut-être de souligner que le racisme à l'égard des Noirs et, dans une moindre mesure, des originaires des pays d'Afrique du Nord, ne doit pas masquer qu'il existait aussi un certain type de racisme de la part des Russes dont étaient victimes les Soviétiques originaires des Républiques asiatiques ${ }^{4}$. Quels qu'aient été en effet les efforts des autorités soviétiques pour fondre en un seul espace étatique les nombreuses entités nationales et ethniques composant l'ex-Union soviétique, dans la conscience collective des Russes, un Bouriate ${ }^{5}$ était — et reste encore dans la Russie d'aujourd'hui — un « citoyen de seconde zone », un « arriéré culturel » qu'il fallait aider à la force du poignet à se hisser au niveau de la civilisation socialiste.

Ainsi, le roman de Zounga Bongolo s'énonce pour l'essentiel comme une dénonciation du racisme de la société soviétique des années 1970, toujours d'actualité dans la Russie de Poutine, en même temps qu'il pose le problème de la difficile coexistence des cultures. Il n'en évoque pas moins en marge les grands thèmes de la propagande officielle : la décadence du monde occidental, la formation d'un homme nouveau, l'âme soviétique, version rénovée de l'âme russe de Soljenitsyn, et lève le voile sur l'une des plaies du système soviétique, le marché noir. Rentré de France avec de la marchandise destinée à la vente au marché noir (blue-jeans et autres vêtements, chaussures, eaux de toilette, produits cosmétiques, etc.), Jan reçoit trois forsovtchik ${ }^{6}$ «vêtus sans élégance de vêtements occidentaux d'une extravagance choquante ». Après avoir débattu des prix, les revendeurs raflent toute la marchandise sans pinailler : un blue-jeans, par exemple, est acheté pour le prix de 220 roubles, soit «le double du salaire d'un ingénieur » (p. 133).

Dans cette histoire qui essaie de transmettre quelque chose de cette vie étudiante en URSS, souvent idéalisée ou abhorrée, l'auteur n'intente aucun procès. Juste révèle-t-il la frontière entre l'idéalité et sa vérité toujours si

4. J.-M. Chauvier, «Un "Kampf" sur fond de crise. Meurtres politiques et racistes en Russie », Le Monde diplomatique, février 2009.

5. Les Bouriates sont des Mongols de Russie et de Mongolie.

6. Des revendeurs au marché noir de produits importés d'Occident. 
relative et nuancée, c'est-à-dire tout simplement humaine. Car là où l'idéalité s'absente, comme dans cette Union soviétique brejnévienne, la tragédie s'inscrit dans les corps : corps du délit épidermique, corps social réduit au simulacre. Même si ce que désigne le corps social n'est plus qu'une grosse machine qui se voudrait au-dessus des contingences humaines mais qui est vite rattrapée par la triste réalité des queues interminables devant les commerces ou la fascination des Soviétiques pour le mode de vie occidental et ses produits de consommation autorisant les spéculations les plus échevelées.

C'est notamment à la lecture de ce quotidien soviétique contrasté que nous invite $\mathrm{Le} \mathrm{Gel,} \mathrm{le} \mathrm{roman} \mathrm{de} \mathrm{Sonallah} \mathrm{Ibrahim.} \mathrm{Lauréat} \mathrm{du} \mathrm{prix} \mathrm{Ibn}$ Rushd $^{7}$ en 2004, Sonallah Ibrahim est l'un des plus grands écrivains vivants de langue arabe. Né en 1937, cet ancien journaliste, militant de gauche, a d'abord connu la prison de 1959 à 1964 avant de se consacrer à la littérature. Tilka l-râ'iha, son premier roman publié en 1966, n'a été traduit en français qu'en 1992 sous le titre Cette odeur-là.

Écrit dans un style nerveux et économe sous la forme d'un journal de bord tenu par un étudiant égyptien boursier de son pays dans les années 1970 à Moscou, Le Gel est un exercice d'autofiction ${ }^{8}$ qui renvoie à sa propre expérience d'étudiant dans une Union soviétique particulièrement marquée par la stagnation sociale après une courte période de dégel sous Nikita Khrouchtchev. C'est l'ère du glacis brejnévien. Le printemps de Prague est passé par là et la société soviétique vit une ère de régression sociale et de pénurie alimentaire particulièrement pénible.

L'auteur de ce journal est un jeune Égyptien, Choukri, doctorant à l'Institut d'Histoire moderne de Moscou dans le cadre des échanges culturels avec l'Union soviétique, qui vit à la Maison des étudiants étrangers où la majorité des résidents est originaire des pays du Tiers-monde. Le roman commence en 1973, année où l'Égypte de Sadate commence à s'éloigner de l'URSS mais où éclate la guerre du Kippour.

Le regard qu'il jette sur la société soviétique de l'époque brejnévienne hantée par la consommation, avec les longues files d'attente devant les magasins et un engouement généralisé pour n'importe quelle pacotille en provenance des pays occidentaux, est plein d'humour, néanmoins sans dénigrement aucun, prenant le parti de ne rien dramatiser. Car dans ce roman où il est peu question de travail ou d'études, c'est à l'analyse du mode de vie de l'homo sovieticus que s'attache avant tout l'auteur. Plusieurs extraits du livre en éclairent les paradoxes et permettent de s'en faire une idée.

7. Plus connu en Occident sous son nom d'Averroès, Ibn Rushd est un philosophe, théologien, juriste et médecin musulman andalou de langue arabe du XII $\mathrm{XI}^{\mathrm{e}}$ siecle. Accusé d'hérésie à la fin de sa vie et bien qu'il n'ait pas eu de postérité immédiate, il reste pour les intellectuels musulmans l'un des plus grands philosophes de la civilisation islamique.

8. C'est ainsi que l'auteur a qualifié ce livre lors d'un entretien accordé à L'Humanité publié le 19 février 2015, <http://www.humanite.fr/un-egyptien-moscou-dans-lesannees-1970-566082>. 
Par exemple au moment où Choukri s'écrie : «Quel drôle de pays, capable d'envoyer des fusées dans l'espace et incapable de fabriquer un rasoir décent pensais-je, en me rasant avec un rasoir soviétique en métal. Ou alors, tout simplement, ils n'en voient pas l'intérêt» (p. 12). Ou encore :

Zoya (Zoya comme Hans plus loin, sont étudiants et amis de Choukri) a proposé qu'on déjeune dans un stolovaya, un genre de cafétéria bon marché où l'on mange pour moins de trois roubles. Le menu consistait en une salade et une soupe de poisson, suivies d'une viande, un poisson ou du poulet garni de riz ou de pommes frites, outre le pain, les jus et les fruits. Le tout sous le regard constant du camarade Brejnev. Hans s'est inquiété de la qualité de la nourriture et de l'hygiène. Zoya l'a rassuré : les cuisinières portent des vêtements propres et stérilisés, des bonnets blancs, et tout le personnel est soumis à des visites médicales régulières. La santé de l'homme soviétique est sacrée. Du moins, sa santé physique, a-t-elle ajouté en riant. Pour le mental, c'est une autre affaire (pp. 24-25).

C'est en véritable entomologiste que Sonallah Ibrahim observe ce monde où les étudiants eux-mêmes ne font que manger et boire. Le journal de Choukri décrit avec une certaine insistance l'alcoolisme en milieu étudiant étranger. Cet alcoolisme n'est pas le simple reflet de celui de la société soviétique, expression d'un mal-être existentiel généralisé et d'une grisaille sans nom qui domine les êtres et les choses. Il est aussi la conséquence d'une certaine misère sexuelle en milieu étudiant étranger: alcoolisme et misère sexuelle y sont les deux faces d'une même médaille. Le monde de l'étudiant étranger est un monde confiné, réduit à l'Institut, à la résidence universitaire ou aux «copines soviétiques » que l'on s'échange sans vergogne. Car, paradoxalement, dans le Moscou du glacis brejnévien, s'il y a pénurie alimentaire, il y a en revanche une profusion d'offres sexuelles qui contraste avec les entraves à la liberté sexuelle. De fait, quelles que soient ses prédispositions pour aller vers l'Autre, et son ouverture d'esprit, il est difficile pour l'étudiant étranger de créer des relations conviviales en dehors du milieu qui lui est officiellement assigné, celui de la résidence internationale. «Vous êtes venu en URSS pour faire des études et vous devez rester dans votre milieu »: tel pourrait être l'implicite oukase adressé aux étudiants étrangers pour leur ôter la possibilité de nouer des liens avec le reste de la société.

Et pendant ce temps-là, le spectacle offert par le Moyen et Proche Orient ou encore par l'Égypte sous Anouar El Sadate était au désarroi politique. Aussi le journal de Choukri invite-t-il à démêler le fil des contradictions d'une époque dont les relations de l'Union soviétique avec les régimes autoritaires du Moyen et Proche Orient sont sans doute le symptôme. Il contient, çà et là, des « perles » sous forme de notes sur le climat politique au MoyenOrient en général et en Égypte en particulier qui valent la peine d'être citées pour leur extrême lucidité :

Hamid a demandé à Abas, qui arrivait de Bagdad, des nouvelles d'un étudiant irakien rentré au pays après avoir terminé ses études à l'Institut (d'Histoire moderne de Moscou). 
- Mako, a répondu Abbas.

- Et Abdel-Jabbar?

- Mako aussi.

Je lui ai demandé ce que cela signifiait. Il a passé un doigt le long de son cou sans rien dire.

Il a raconté ensuite l'histoire d'un leader ouvrier arrêté par les baathistes. Après l'avoir dépouillé de ses biens et avoir violé sa femme, ils l'ont contraint à avoir des rapports sexuels avec un jeune garçon tandis qu'ils prenaient des photos, puis ils l'ont menacé de diffuser les photos auprès de ses camarades ouvriers. À la fin, il s'est plié à leurs exigences. [...]

Je l'interroge :

- Et ce complot que la presse soviétique présente comme une machination impérialiste contre le régime baathiste ?

- Du pipeau. Le Baath fait la chasse aux nassériens avec la bénédiction de l'Union soviétique.

À la demande de Hamid, il a aussi raconté comment (les barbouzes du Baath) ont essayé d'assassiner le leader kurde Barzani et comment ils ont fait avouer Azizel-Hadj, le chef du parti communiste dissident. Ils ont exécuté sous ses yeux plusieurs de ses compagnons l'un après l'autre après les avoir mutilés, en particulier les parties génitales (pp. 40-41).

La barbarie moyenâgeuse des nervis du Baath soutenue par l'Union soviétique devient le révélateur de cette politique d'alliance du PCUS (Parti communiste de l'Union soviétique) avec «les forces nationalistes, anti impérialistes et progressistes du Tiers-monde », dont les premières victimes furent les communistes et les militants arabes de gauche.

En citant dans son journal cet extrait d'un roman de l'écrivain américain Thornton Wilder : «Aucun gouvernement n'est en mesure d'offrir aux hommes ce qui assurerait leur bonheur, la discorde étant au cœur du monde, sous tous les cieux» (p. 70), Sonallah Ibrahim pourrait bien laisser entendre que la société socialiste, au sens où la concevaient Marx et les révolutionnaires russes qui renversèrent le régime tsariste, relèverait de l'irréalisable ou de l'imaginaire.

Esprit curieux et fin observateur de son environnement, Choukri est un homme de réflexion ouvert sur l'Autre et sur son temps, qui tente de se construire une vision substantielle propre et réaliste de l'Homme. D'où la fréquentation, dans le texte, des écrivains de son pays dont le grand romancier Naguib Mahfouz ou des compositeurs comme Rimski-Korsakov, Chostakovitch, Rossini qu'il va écouter au Bolchoï. C'est aussi un homme de réflexion et de conviction pour qui le bonheur terrestre in fine n'est qu'une utopie.

Tout comme Amrikanli, cet autre grand livre de Sonallah Ibrahim, où apparaît déjà Choukri en universitaire égyptien, professeur d'histoire en poste à San Francisco, Le Gel paru en arabe au Caire, en 2011, fait partie de ces productions littéraires qui, vingt-deux ans après la chute du mur de Berlin, relancent le débat sur la nature idéologique du régime soviétique et des États de l'Est européen. Comme Zounga Bongolo, mais probablement pas pour les mêmes raisons, Sonallah Ibrahim a attendu trente ans avant 
de publier son roman, dont il avait rassemblé les matériaux dès les années 1980. Après les mésaventures intérieures de «l'Internationalisme prolétarien » et son racisme quotidien décrit par Zounga Bongolo, Sonallah Ibrahim vient enrichir la critique lucide et sereine sous la forme littéraire, de ce que certains auteurs marxistes critiques ${ }^{9}$ ont dénommé le «socialisme existant réellement ».

Association Rupture, Brazzaville (République du Congo).

\section{RÉSUMÉ}

Deux romans écrits avec en arrière-plan l'expérience de leurs auteurs, anciens étudiants en URSS pendant la période brejnévienne, mettent au jour les contradictions internes de la société soviétique et l'échec du «socialisme réellement existant ». Dans Un Africain dans un iceberg, Zounga Bongolo dénonce le racisme ordinaire de la société soviétique, à travers l'histoire $d^{\prime}$ 'un amour impossible entre une jeune étudiante russe et un étudiant originaire du Congo-Brazzaville. Le grand écrivain Sonallah Ibrahim compose dans un style très sobre un exercice d'autofiction avec le journal de bord de Choukri, étudiant égyptien qui observe les échecs et les tares du système soviétique à travers la vie quotidienne de ses camarades internationaux, tout en évoquant la situation politique de l'Égypte, alors en pleine guerre du Kippour.

\section{ABSTRACT}

Voyage to Brezhnevland. The Dream Life of Third World Students in Soviet Russia. - Two novels written against the backdrop of their authors' experiences in the USSR during the Brezhnev era bring to light internal contradictions in Soviet society and the failure of "real life Socialism". In An African in an Iceberg, Zounga Bongolo denounces everyday racism in Soviet society in recounting an impossible love story between two students, a young man from Congo-Brazzaville studying in the USSR and a young woman studying at the same Russian university. The celebrated writer, Sonallah Ibrahim, composes a sober stylistic exercise in autobiographical fiction with Choukri's diary in which the Egyptian student observes the failures and dysfunctions of the Soviet system in the daily lives of his international classmates whilst making reference to the political situation in Egypt during the Yom Kippur War.

Mots-clés/Keywords: URSS, autofiction, étudiants, expérience, littérature africaine, racisme, roman/USSR, autobiographical fiction, student, experience, African literature, racism, novel.

9. Voir notamment R. BAHRO, L'alternative : Pour une critique du socialisme existant réellement, Paris, Stock 2, 1979 ; L. Plouchtch, Dans le carnaval de l'histoire. Mémoires, Paris, Éditions du Seuil, 1977. 